\title{
ANÁLISIS DE LOS FACTORES QUE INCIDEN EN LA DESESTABILIZACIÓN DE LAS COLUMNAS DE LA CATEDRAL DE SAL EN ZIPAQUIRÁ, CUNDINAMARCA
}

\author{
(Analysis of the factors that have an impact on the destabilization of the columns of the Sal \\ Cathedral of Zipaquirá, Cundinamarca)
}

\author{
Diana Rocío Galvis Botía*, Nydia Genith Peñaranda* Jaime William Jojoa Muñoz **
}

* Escuela de Ingeniería de Minas, Uptc. Grupo inv. SFSS. **Escuela de Ingeniería de Minas, Uptc. Grupo inv. GEAM, jaime.jojoa@uptc.edu.com

(Recibido septiembre 23 de 2014 y aceptado noviembre 12 de 2014)

\begin{abstract}
Resumen:
Este artículo analiza los factores que generan mayor afectación en la estabilidad de las cámaras 360,380 y 400, que constituyen el espacio ocupado por la Catedral de Sal de Zipaquirá, mediante el estudio de las propiedades tanto físicas como mecánicas de la matriz rocosa. A partir de allí se determinan las propiedades de macizo y su posible comportamiento ante el estado de esfuerzos verticales y horizontales, además de las cargas actuantes sobre machones y columnas, teniendo en cuenta las condiciones de los planos de discontinuidades presentes en los elementos de sostenimiento natural. Posteriormente se analizan algunos de los factores que generan la mayor inestabilidad para definir cuál de ellos incide de manera dominante y determinar un método para mitigarlo.
\end{abstract}

Palabras clave: estabilidad, resistencia, esfuerzos, columna, pilar, discontinuidad.

\section{INTRODUCCIÓN}

La Catedral de Sal de Zipaquirá es considerada uno de los logros arquitectónicos y artísticos más notables de la actualidad. El espacio ocupado por esta catedral es parte del nivel Fabricalta, uno de los niveles antiguamente explotados del depósito salino, cuya recuperación se realizaba por el método de cámaras largas, pero actualmente debido al lento proceso de deterioro y al tiempo, los machones en cuyo lugar se

\section{Abstract:}

This article examines the factors that generate greater involvement in the stability of the chambers 360,380 and 400 which constitute the space occupied by the Salt Cathedral of Zipaquirá, through the study of the physical and mechanical properties of the rock matrix. Thereafter we determine the solid properties and their possible behavior under vertical and horizontal stress state, as well as the loads acting on piers and columns, taking into account the conditions of the planes of discontinuities present in the elements of natural sustaining. Then we discuss some of the factors that generate the greatest instability to define which one is the most dominant and determine a method to mitigate it.

Keywords: stability, strength, efforts, column, pillar, discontinuity

diseñaron cuatro columnas cilíndricas que simbolizan los cuatro evangelistas, han venido presentando indicios de desestabilización, representados principalmente en la presencia de fisuras que pueden generar caída de bloques ocasionando gran inseguridad. Por lo anterior, el objetivo del presente artículo es analizar minuciosamente los factores que posiblemente generan esta inestabilidad, para mejorar las condiciones de las cámaras y así aumentar la vida útil de la Catedral. 
Esta investigación se enfoca en determinar los factores que inciden en la desestabilización de las columnas, machones y techos de las cámaras que conforman la Catedral de Sal de Zipaquirá, Cundinamarca, Colombia. Para lo cual se definieron como objetivos dentro de la investigación los siguientes: i) Obtener las propiedades físico-mecánicas del macizo rocoso, ii) Realizar el análisis estructural de acuerdo con las condiciones de las discontinuidades, iii) Definir el estado de los esfuerzos actuantes sobre el área de estudio, iv) Determinar la resistencia y la estabilidad de los machones y columnas de la catedral de Sal., v) Evaluar los resultados del análisis de las propiedades físico-mecánicas, estado de esfuerzos, así como discontinuidades para determinar el grado de incidencia e importancia en la desestabilización; y vi) Analizar las diferentes alternativas de sostenimiento.

\section{LOCALIZACIÓN GEOGRÁFICA}

El yacimiento de sal de Zipaquirá está situado aproximadamente a $1 \mathrm{~km}$. al occidente de la ciudad del mismo nombre, a uno $49 \mathrm{~km}$. al norte de la ciudad de Bogotá, D.C. El contrato de concesión HIQO - 03 tiene un área de 337hás $4717 \mathrm{~m}^{2}$ y dentro del área de concesión minera se encuentra el denominado Parque de Sal, con un área de 23 hás; dentro de esta área se encuentra la Catedral de Sal de Zipaquirá. El presente estudio se centra en las cámaras centrales 360, 380 y 400.

\section{CARACTERIZACIÓN GEOMECÁNICA}

\subsection{Geología del área de estudio}

En el área se presentan cuatro tipos de sal:

Horizonte salino S1 (halita de alta pureza). Bandas de halita blanca a gris según impurezas arcillosas.

Horizonte salino S2 (masivo con clastos o melange fino). Roca masiva de estratificación grotesca, compuesta de bloques clásticos de lutita arcillosa.

Horizonte salino S3 (brechas sedimentarias o melange grueso). Estratos o lentes de conglomerados brechosas con bloques de lutita arcillosa negra hasta de $5 \mathrm{~m}$ de diámetro con estratificación errática grotesca dentro de una matriz arcillosa salina.
Horizonte salino S4 (lutitas). Roca detrítica arcillosa de grano muy fino, estratificación fina a laminar y color negro a gris.

En el área se evidencian los cuatro tipos y se caracteriza como más representativo el horizonte S2, así:

Roca claramente estratificada con planos de estratificación de cristales de sal ligeramente alargados, donde se alternan sustancias arcillosas asociadas de anhidrita $\mathrm{CaCo}_{4}$ y polihalita $\left(\mathrm{K}_{2} \mathrm{Ca}_{2} \mathrm{Mg}\right)\left(\mathrm{CO}_{4} 2 \mathrm{~h}_{2} \mathrm{O}\right)$, y capas laminares de sal de roca con espesores muy variables. El contenido de insolubles es del 10 al $12 \%$.

\subsection{Análisis de discontinuidades}

El objetivo del análisis de discontinuidades es determinar las características de las mismas y su influencia sobre las columnas y machones que constituyen el área de estudio, para lo cual se realizó un levantamiento de 503 discontinuidades, distribuidas equitativamente en las paredes de las cámaras 360, 380 y 400 . El análisis de discontinuidades se llevó a cabo por medio del programa Dips 5.1.

Se definieron tres familias de discontinuidad, dos de diaclasas y una de estratificación, con orientaciones 80/168, 80/30 y 13/104 estratificación (ver figura 1).

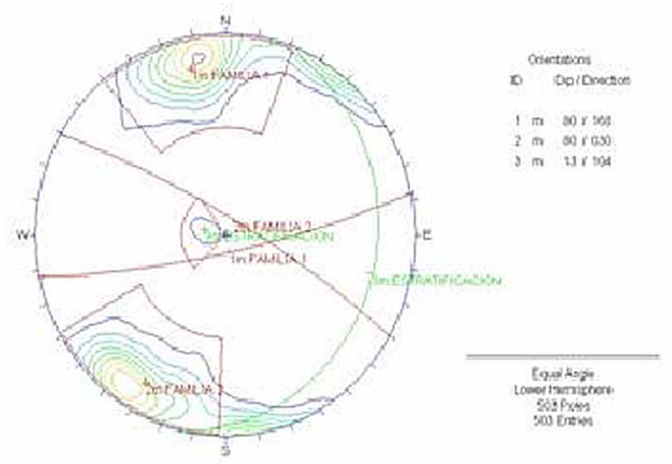

Figura 1. Orientación de familias predominantes. Resultados estudio, análisis de datos programa Dips.

Se definen también otros parámetros como:

Espaciamento: se determinó que un $50 \%$ de discontinuidades presenta un espaciamiento entre 200$600 \mathrm{~mm}$, esto las clasifica como moderadamente unidas.

Rugosidad: un $51 \%$ de las discontinuidades presenta una 
rugosidad tipo IV, lo cual las clasifica como onduladas rugosas, JRC entre 6-8.

Abertura: se definió que el $52 \%$ de las discontinuidades presenta una abertura entre $0.5-2.5 \mathrm{~mm}$ (baja abertura).

Continuidad en el rumbo: un $51 \%$ de las discontinuidades presenta una continuidad en el rumbo entre 1-3 m (continuidad baja).

Continuidad en el buzamiento: un $48 \%$ de las discontinuidades presenta una continuidad entre 1-3 m.

\subsection{1 Ángulo de ficción residual de discontinuidades.}

Este ángulo se determina mediante el ensayo Tilt Test. Para este ensayo se usaron bloques de roca que contengan planos de discontinuidad sin cohesión, los bloques fueron colocados sobre una superficie de apoyo, la cual se inclinó hasta que se produjo un deslizamiento de un fragmento sobre el otro. Cuando se inició el desplazamiento se midió el ángulo $\left(\beta=\emptyset_{b}\right)$ que forma la superficie de apoyo con la horizontal.

Aplicando la expresión propuesta por Barton y Choubey, es posible determinar el ángulo de fricción residual a través de la Ecuación (1).

$$
\emptyset_{r}=\left(\emptyset_{b}-20^{\circ}\right)+20 \frac{r}{R}
$$

Donde, $\beta$ : ángulo que genera el deslizamiento de un bloque sobre otro; $\emptyset b$ : ángulo de fricción básico (Tilt test); $R$ : valor del rebote del martillo Schmidt (roca sana) y $r$ : valor del rebote del martillo Schmidt (sobre discontinuidad).

Como las paredes de las discontinuidades están sanas $R=r$.

Tabla 1. Ángulos de fricción residuales de discontinuidades

\begin{tabular}{cc}
\hline Muestra & Angulo fricción básico $(\varnothing b)$ \\
\hline 1 & 40 \\
2 & 36 \\
3 & 42 \\
4 & 34 \\
5 & 38 \\
Promedio & $\mathbf{3 8}$ \\
\hline
\end{tabular}

Fuente: resultados de estudio.

\subsubsection{Orientación de esfuerzos tectónicos residuales.}

Para determinar dichas orientaciones, es necesario seleccionar las dos orientaciones de familias de discontinuidades más importantes del área estudiada y proceder de la siguiente manera:

La intersección de los dos círculos mayores corresponde a $\sigma_{2}$.

La bisectriz del ángulo agudo, comprendido entre las discontinuidades del círculo máximo $\sigma_{1}-\sigma_{3}$ sitúa a $\sigma_{3}$. A $90^{\circ}$ de $\sigma_{3}$ en el círculo máximo $\sigma_{1}-\sigma_{3}$ se ubica $\sigma_{1}$, $\sigma_{1}=10 / 80, \sigma_{2}=67 / 102, \sigma_{3}=26 / 280$., Ver Figura 2 y 3 .

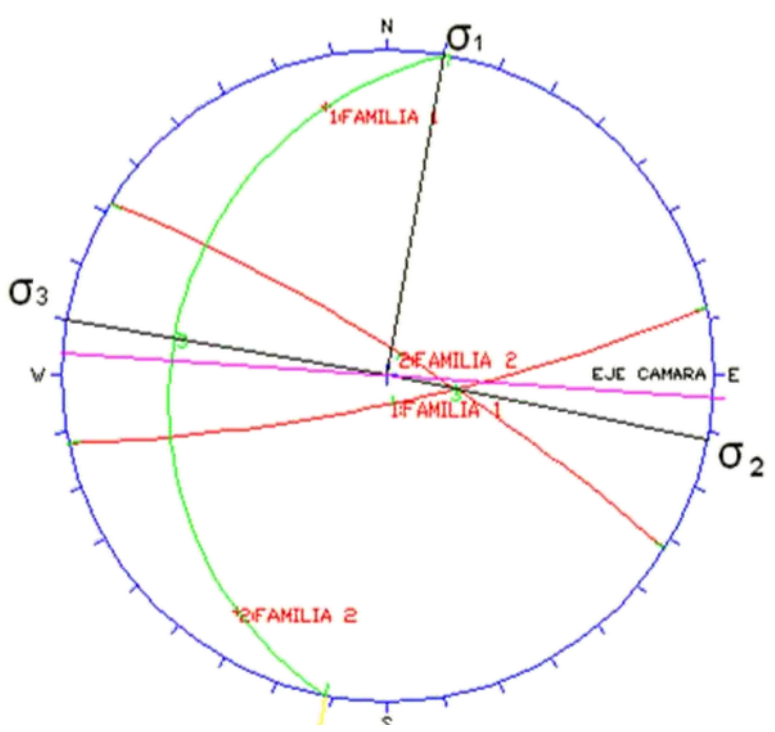

Figura 2. Orientación de esfuerzos tectónicos residuales a partir de análisis estereográfico. Resultados estudio, análisis de datos programa Dips.

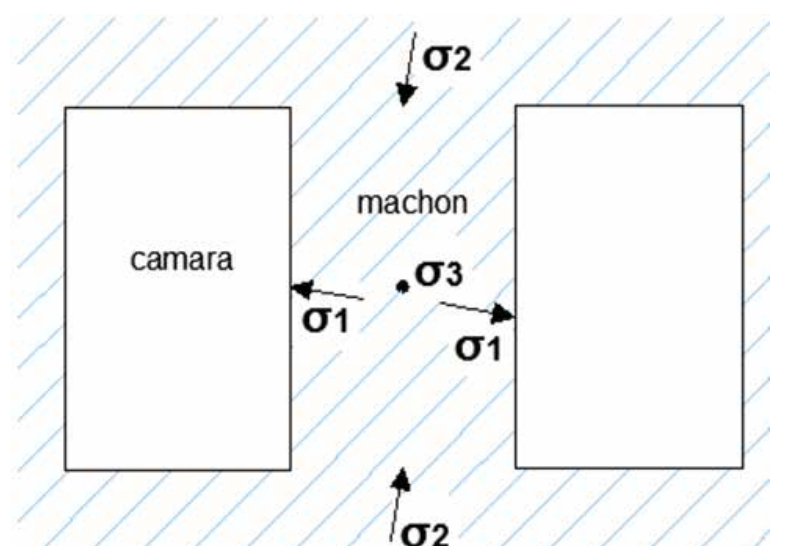


Figura 3. Orientación de esfuerzos tectónicos residuales respecto a la sección de la cámara.

Se determina que $\sigma_{2}$ actúa de manera vertical generando baja concentración de esfuerzos en el techo, pero el esfuerzo principal mayor actúa casi perpendicular al eje de la cámara, de manera desfavorable sobre la sección de la excavación, lo que puede ser una de las causas que favorecen la abertura de diaclasas en los machones.

\subsubsection{Diagrama de bloques generados.}

Se definieron tres familias de discontinuidades predominantes. La estratificación, debido a sus condiciones dentro del macizo, no representa un plano de debilidad.

Como se observa en la Figura 4, los bloques formados en las paredes de las cámaras son alargados y presentan una continuidad de techo a piso, con variación en su tamaño.
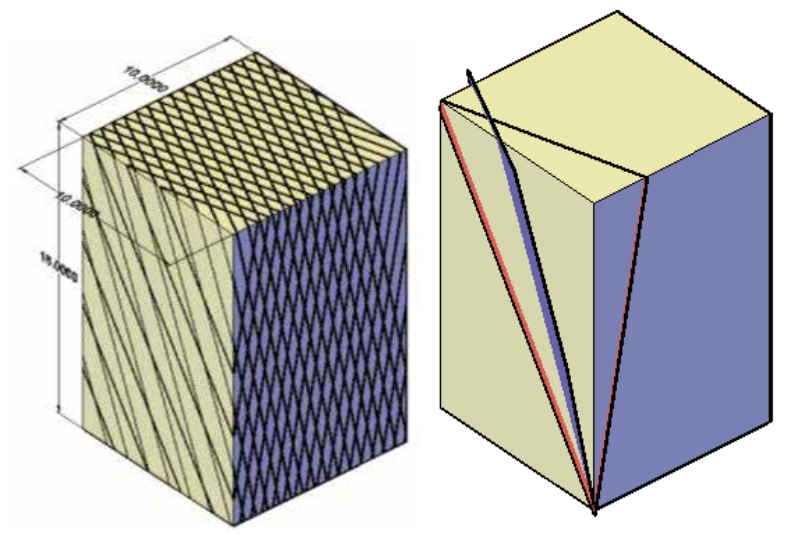

Figura 4. Diagrama de bloques.

Fuente: resultados del estudio.

\subsection{Propiedades fisicas}

Para definir estas propiedades, se tomaron 10 muestras tamaño puño, distribuidas de manera aleatoria y se obtuvieron como resultados un peso específico en condición natural de $22,05 \mathrm{KN} / \mathrm{m}^{3}$, en condición seca $22,03 \mathrm{KN} / \mathrm{m}^{3}$ y una humedad natural de $0,07 \%$ muy baja.

\subsection{Propiedades mecánicas}

\subsubsection{Resistencia a compresión simple.}

La población de estudio corresponde a 25 probetas de roca tipo halita de 2 pulgadas de diámetro por 4 pulgadas de longitud, con una relación de esbeltez de 2 (ver Fotografía 1), clasificada en tres grupos de muestras. El primer grupo de muestras posee Halita en un $70 \%$ en peso, el segundo un $80 \%$ y el otro grupo $90 \%$.

Las muestras falladas presentaron un fenómeno de barril (ver Fotografía 2), es decir, las probetas mostraron gran deformación diametral.

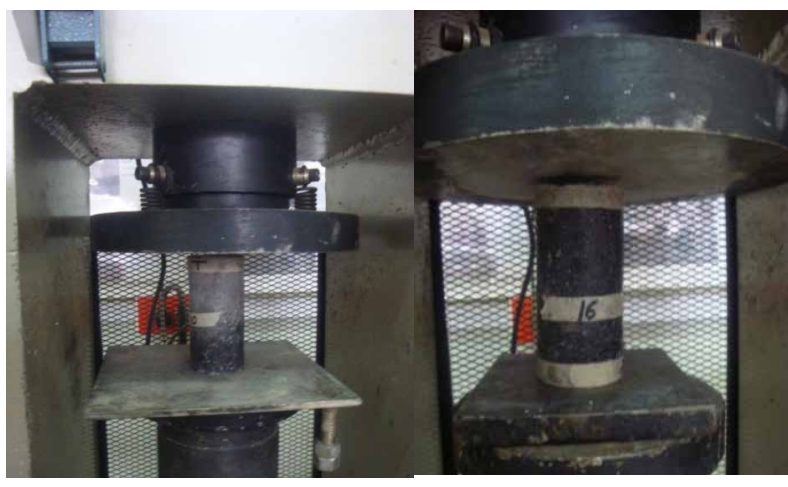

Fotografía 1. Probetas cilíndricas sometidas a compresión simple.

De acuerdo con el histograma de frecuencia (ver Figura 5), se tiene una curva asimétrica bimodal con un media ponderada de 22,02 MPa y una desviación estándar de $\pm 4,29$.

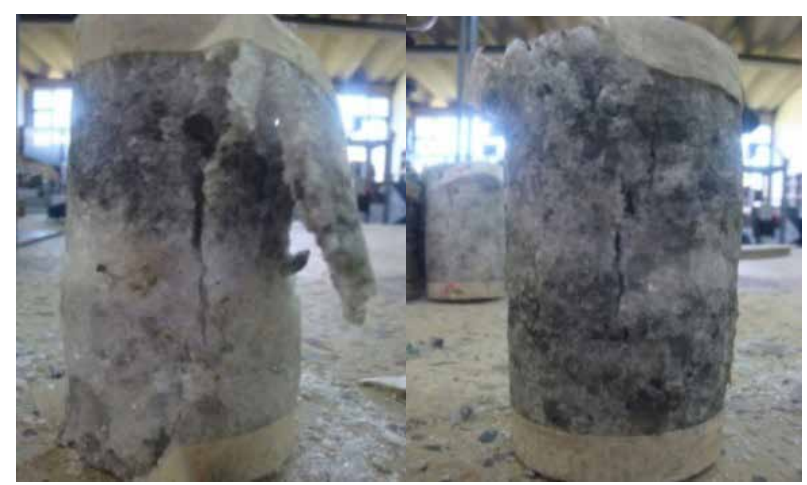

Fotografía 2. Falla generada en las probetas al aplicar carga axial no confinada. 


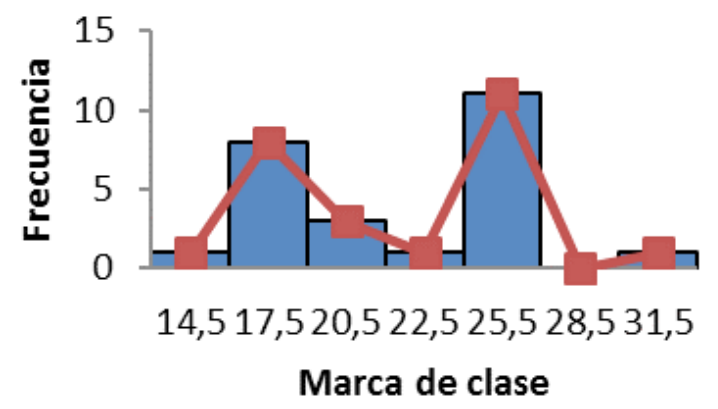

Figura 5. Histograma y polígono de frecuencias para resistencia a compresión simple.

Se define un coeficiente de variación de 0,19 , que equivale aproximadamente al $20 \%$, lo que indica que hay una variabilidad media en los datos.

Para determinar la curva de comportamiento inicialmente se identifica a qué tipo de función se asemeja cada una de las curvas, obteniéndo que la función que presenta una mayor precisión es un polinomio de tercer o cuarto grado, ver expresiones (3) y (4).

$$
\begin{gathered}
f(x)=a x^{4}+b x^{3}+c x^{2}+d x+e \text { grado } \\
f(x)=b x^{3}+c x^{2}+d x+e 3 \text { grado }
\end{gathered}
$$

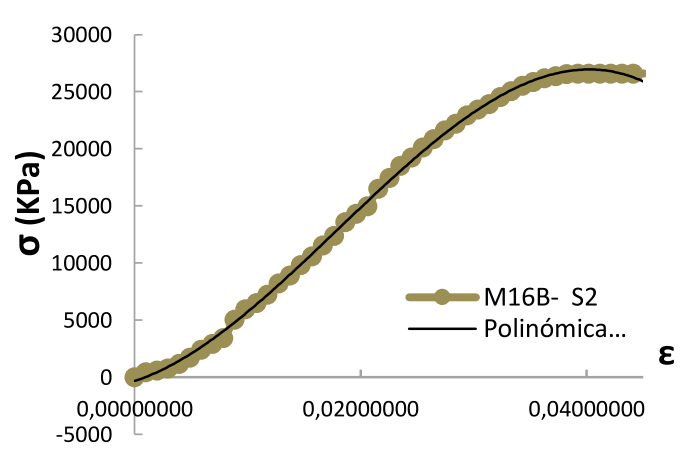

Figura 6. Función de la curva para compresión simple de sal (tercer orden).

$y=-6 \times 10^{8} x^{3}+3 \times 10^{7} x^{2}+3,3 \times 10^{5} x-331,76$ $\mathrm{R}^{2}=0,9992$

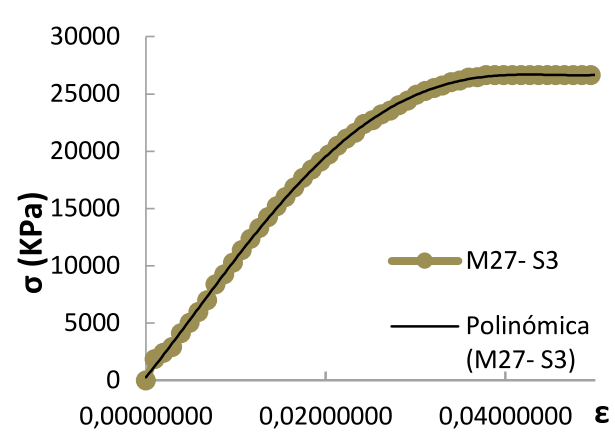

Figura 7. Función de la curva para compresión simple de sal (cuarto orden).

$y=8 \times 10^{9} x^{4}-8 \times 10^{8} x^{3}+1 \times 10^{7} x^{2}+1 \times 10^{6} x+246,18$

$\mathrm{R}^{2}=0,9997$

Como se aprecia en las Figuras 6 y 7, las curvas se caracterizan por una parte inicial cóncava hacia arriba, que se hace más pendiente al cerrarse las superficies de micro fisuras existentes en la roca, seguida por una sección recta propia del comportamiento elástico; posteriormente se muestra una deformación inelástica creciente y una fluencia continua.

De las curvas se aprecia como al terminar la etapa elástica, la deformación sigue aumentando sin que se pierda la resistencia en cada una de las curvas. Esto significa que la resistencia se mantiene constante después de grandes deformaciones, en este caso la resistencia pico y la resistencia residual son iguales, por lo tanto se define que la roca presenta un comportamiento elastoplástico dúctil.

\subsubsection{Módulo de elasticidad.}

El módulo de elasticidad o módulo de Young de la roca fue determinado a partir de las curvas de esfuerzo deformación obtenidas en el ensayo de compresión simple, y el método empleado para su cálculo fue el del módulo medio o pendiente de la porción recta (ver expresión 5).

$$
\mathrm{E}=\frac{\Delta \sigma}{\Delta \varepsilon}
$$

Donde,

E: módulo de elasticidad 
$\Delta \sigma: \quad$ variación de esfuerzo.

$\Delta \varepsilon: \quad$ variación de deformación.

Se calcula una media ponderada de 1054, $54 \mathrm{Mpa}$, con una desviación de $\pm 242,07$, con un coeficiente de variación de 0,24 equivalente al $24 \%$, lo que indica una dispersión media en los datos, (Ver Figura 8).

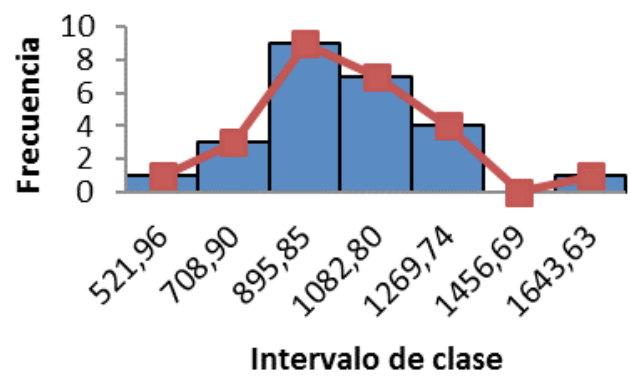

Figura 8. Histograma y polígono de frecuencia Módulo de elasticidad.

Resistencia a compresión simple mediante el uso del martillo Schmidt. Para hallar la resistencia mediante este ensayo se calcula el valor del rebote medio con los datos de campo. Para ello, de cada 10 valores se eliminan los cinco menores y se obtiene el valor medio con los cinco restantes, con este valor y el peso específico se aplica la ecuación (6).

$$
\log J C S=0,00088 \gamma_{\text {roca }} r+1,01
$$

Donde, JCS es la resistencia a compresión de las paredes de la discontinuidad (MPa), y $\gamma_{\text {roca }}$ es el peso específico de la roca $\left(\mathrm{KN} / \mathrm{m}^{3}\right)=22 \mathrm{KN} / \mathrm{m}^{3}$. Para este ensayo se tomaron 40 medidas en roca intacta y 30 medidas de resistencia de discontinuidades en cada pared del machón que conforman las cámaras, y entre 20 a 30 medidas alrededor de las columnas circulares. En las Tablas 2 y 3 se presentan los datos medidos a través de este ensayo.

Tabla 2. Datos medidos sobre machones en roca sana y discontinuidades mediante martillo Schmidt.

$\begin{array}{lcccc} & \begin{array}{c}\text { MACHÓ } \\ \text { N 1 }\end{array} & \begin{array}{c}\text { MACHÓ } \\ \text { N 2 }\end{array} & \begin{array}{c}\text { MACHÓ } \\ \mathbf{N ~ 3}\end{array} & \begin{array}{c}\text { MACHÓ } \\ \text { N 4 }\end{array} \\ \begin{array}{l}\text { Promedio rebote } \\ \text { roca sana }\end{array} & 2,1 & 1,9 & 1,9 & 2,0 \\ \begin{array}{l}\text { Promedio rebote } \\ \text { discontinuidades }\end{array} & 1,8 & 1,8 & 1,8 & 1,9 \\ \begin{array}{l}\sigma_{\mathrm{ci}}(\mathrm{MPa}) \text { roca } \\ \text { sana }\end{array} & 11,23 & 11,15 & 11,13 & 11,17 \\ \begin{array}{l}\sigma_{\mathrm{ci}}(\mathrm{MPa}) \\ \text { discontinuidades }\end{array} & 11,09 & 11,10 & 11,8 & 11,14\end{array}$

Fuente: resultados de estudio.

Tabla 3. Datos tomados sobre columnas circulares mediante martillo Schmidt

\begin{tabular}{ccccc}
\hline & COLUMNa 1 & COLUMNa 2 & COLUMNa 3 & COLUMNA 4 \\
\hline Promedio & 1,8 & 1,5 & 1,8 & 1,7 \\
$\sigma_{\mathrm{ci}}(\mathrm{MPa})$ & 11,09 & 10,95 & 11,11 & 11,02 \\
\hline
\end{tabular}

Fuente: resultados de estudio.

Como se observa, los valores son bastante inferiores en comparación con los obtenidos en el ensayo de resistencia a compresión simple.

3.5 Propiedades dinámicas elásticas de la roca a bajos niveles de deformación.

Para determinar las velocidades de propagación de onda necesarias para el cálculo de las propiedades dinámicas de la roca (módulo de elasticidad, módulo de rigidez y módulo de Bulk) se usó el equipo GCTS Testing Systems CATS Ultrasonics 1,83 a 19 muestras cilíndricas de aproximadamente 2 " de diámetro y 4 " de alto.

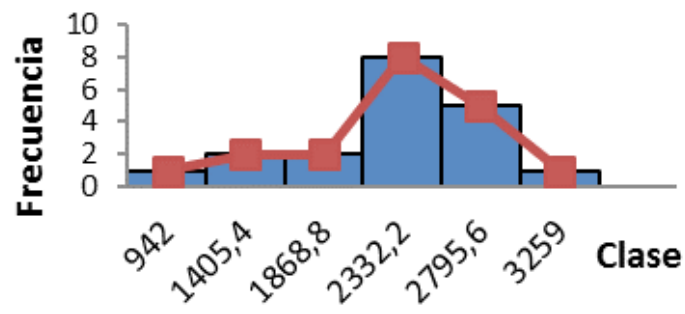

a) Onda $P$ 


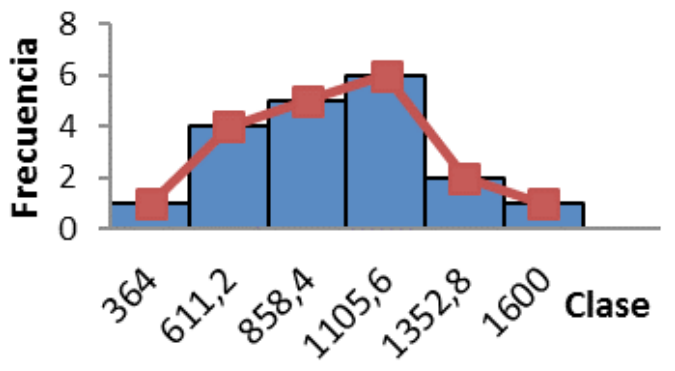

b) Onda $\mathrm{S}$

Figura 9. Histograma de frecuencias velocidad de onda P (superior) y $\mathrm{S}$ (inferior).

Las distribuciones tanto de la onda $\mathrm{P}$ como la $\mathrm{S}$ son simétricas sesgadas a la izquierda, (ver Figura 9). La onda $\mathrm{P}$ con un promedio ponderado de 2287,42 y una desviación estándar de $\pm 595,52$ y la onda $\mathrm{S}$ una media de 855,05 y una desviación de $\pm 308,57$.

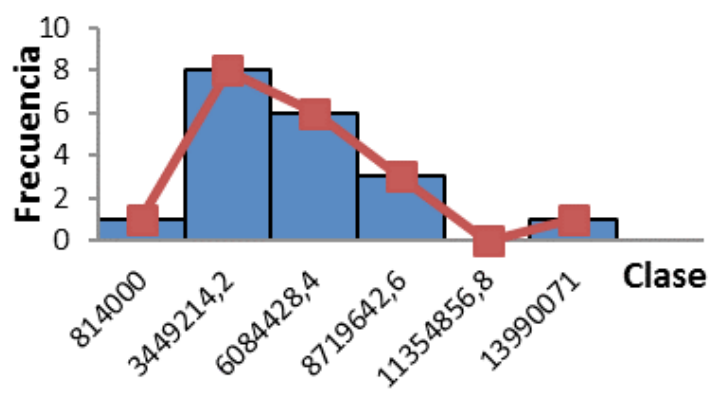

a). Módulo de Young

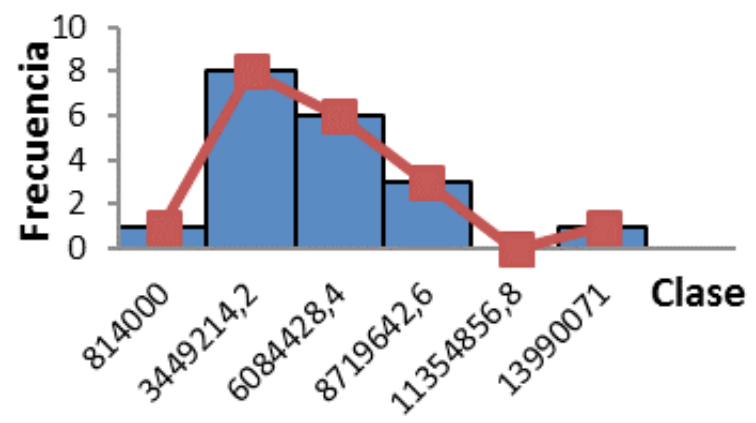

b) Módulo de Bulk.

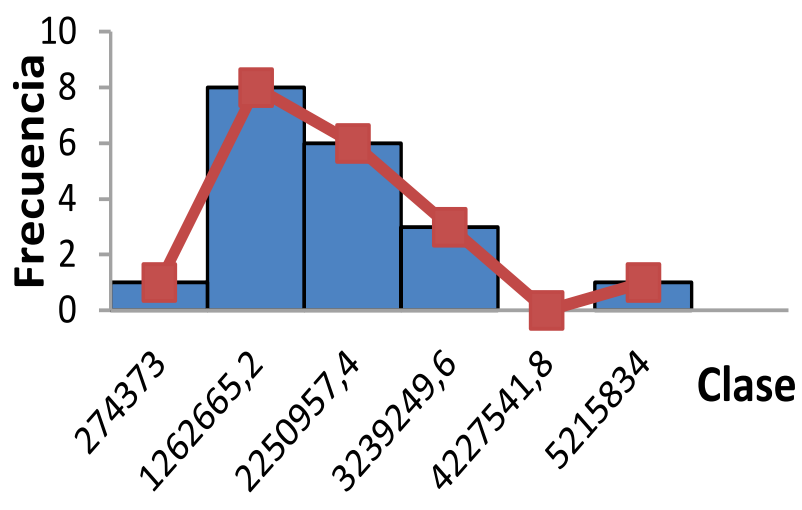

c) Módulo de rigidez por cortante

Figura 10. Histogramas de frecuencias módulos dinámicos de la roca. (a) Módulo de Young. (b) Módulo de Bulk. (c) Módulo de rigidez por cortante.

La Figura 10 muestra cómo los tres histogramas presentados son simétricos sesgados a la derecha. Para el i) Módulo de Young se tiene una media ponderada de 4642,00 y una MPa desviación de $\pm 3175,57$; para el ii) Módulo de Bulk la media ponderada es de 7330,69 y la MPa desviación de $\pm 3548,60$, y para iii) el Módulo de rigidez la media ponderada es 1686,69 y la MPa desviación de $\pm 1183,16$.

\subsection{Clasificación geomecánica MRMR (Mining Rock Mass Rating)}

Para este caso se usó la clasificación del macizo rocoso (MRMR) (Hoek,1980), diseñada por Bienawsky en 1989, para definir la calidad de la roca.

El Índice de calidad de la Roca RQD (Rock Quality Designation) (Hoek, 1980, p. 43), indica el grado de fracturación del macizo rocoso, se usó la propuesta en función del análisis de paredes de los machones y se determinó a partir de la frecuencia de discontinuidades en una longitud de $5 \mathrm{~m}$. La expresión para calcular el RQD se presenta en la ecuación (7). En la Tabla 4 se presentan los resultados del índice RQD calculados para cada machón de estudio.

$$
R Q D=100 * e^{-0.11} *(0,1 \lambda+1)
$$

Donde, $\lambda$ : es la Frecuencia de discontinuidades.

$$
\lambda=\frac{\text { No de discontinuidades }}{\text { Longitud medida }}
$$


Tabla 4. Cálculo del RQD para cada machón en estudio.

\begin{tabular}{ccccc}
\hline Machón & $\begin{array}{c}\text { No. de } \\
\text { Fracturas }\end{array}$ & $\begin{array}{c}\text { Longitud } \\
\text { medida }\end{array}$ & $\boldsymbol{\lambda}$ & RQD \\
(Fracturas/m) & \\
\hline 1 & 8 & $5 \mathrm{~m}$ & 1,6 & 98,8492 \\
2 & 11 & $5 \mathrm{~m}$ & 2,2 & 97,9079 \\
2 & 10 & $5 \mathrm{~m}$ & 2,0 & 98,2483 \\
3 & 9 & $5 \mathrm{~m}$ & 1,8 & 98,5624 \\
3 & 9 & $5 \mathrm{~m}$ & 1,8 & 98,5624 \\
4 & 10 & $5 \mathrm{~m}$ & 2,0 & 98,2483 \\
& & \multicolumn{3}{c}{ Promedio } \\
\cline { 2 - 5 }
\end{tabular}

\subsubsection{RQD perforación columna 2.}

El índice RQD puede medirse a través del análisis de la perforación realizada en la columna 2 en dirección W- E y se determina mediante la expresión (9).

$$
\begin{gathered}
R Q D=\frac{\text { Elong } \cdot \text { trozos perforacion }>10 \mathrm{~cm}}{\text { longitud total de la perforacion }} \\
* 100(9)
\end{gathered}
$$

A través de las mediciones realizadas se define que existe entre las secciones extraídas de perforación que superan los $10 \mathrm{~cm}$ un total de 7.57 y una longitud de perforación de $8 \mathrm{~m}$. Obteniendo un RQD=94,62, lo cual califica la roca como de buena calidad.

3.6.2 Resultados clasificación MRMR. Los puntajes de la clasificación geomecánica MRMR, se presentan a continuación:

Resistencia de la roca intacta 2

Rock Quality Designation (RQD)

Espaciado de discontinuidades

Condición de discontinuidades

Condiciones de agua

Puntuación parcial

Ajuste por orientación de discontinuidades

Puntaje Mining Rock Mass

Rating (MRMR)

Esto clasifica la roca clase II (buena calidad), aunque se encuentra dentro del límite mínimo de rango de clasificación, por lo tanto tiene calidad entre regular y buena.

\subsection{Curvas de equilibrio límite}

Las curvas de equilibrio del macizo rocoso se determinan por medio del criterio generalizado de Hoek y Brown, el cual se expresa como (Hoek, Carranza \& Corkum, 2002, p. 3) se indica en (10).

$$
\sigma_{1}=\sigma_{3}+\sigma_{c i}\left(m_{b} \frac{\sigma_{3}}{\sigma_{c i}}+s\right)^{a}
$$

Con la aplicación a túneles se obtuvieron los resultados reportados en la Tabla 6 y en la Figura 11.

Tabla 5. Propiedades mecánicas del macizo rocoso obtenidas a través del Roclab.

\begin{tabular}{lc}
\hline \multicolumn{1}{c}{ Propiedad } & valor \\
\hline $\mathrm{Mb}$ & 0,382 \\
$\mathrm{~S}$ & 0,0020 \\
$\mathrm{~A}$ & 0,504 \\
Cohesión $(\mathrm{C})$ & $0,273 \mathrm{MPa}$ \\
Angulo de fricción $(\emptyset)$ & $32,78^{\circ}$ \\
Resistencia a tensión $\left(\sigma_{t}\right)$ & $-0,113 \mathrm{MPa}$ \\
Resistencia a compresión $\left(\sigma_{c m}\right)$ & $0,955 \mathrm{MPa}$ \\
Resistencia global & $1,909 \mathrm{MPa}$ \\
Módulo de elasticidad $\left(E_{m}\right)$ & 167,33 \\
\hline
\end{tabular}

Fuente: resultados análisis con programa Roclab.

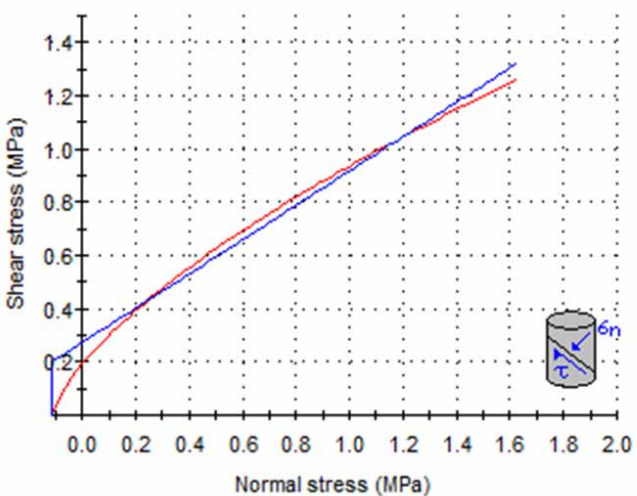

Figura 11. Envolventes de resistencia del macizo rocoso Criterio generalizado de Hoek- Brown (2002) y Mohr - Coulomb. Resultados análisis con programa Roclab.

La relación entre el esfuerzo vertical y horizontal $11 K=\frac{\sigma_{3}}{\sigma_{1}}$. 
Por lo tanto, conociendo que la excavación está a una profundidad de aproximadamente $80 \mathrm{~m} \sigma_{1}=1,64 \mathrm{MPa}$; por RocLab $\sigma_{3}=0,16$; entonces, $K=\mathbf{0 , 1 2}$.

\section{ANÁLISIS DEL ESTADO TENSIONAL}

\subsection{Esfuerzos naturales}

Para el caso del cálculo de esfuerzos naturales en macizos rocosos suele apoyarse en el cálculo de los esfuerzos gravitacionales, donde esfuerzo vertical en un punto es debido a la carga de materiales suprayacentes, que viene dada por (12).

$$
\sigma_{v}=\gamma h(12)
$$

Donde, $\sigma_{v}$ : esfuerzo vertical, $\gamma$ corresponde al peso específico de la roca suprayacente y $h$ : es el espesor de las capas de recubrimiento.

$$
\sigma_{h}=K \sigma_{v}
$$

A la profundidad de las cámaras se presenta un recubrimiento de $39 \mathrm{~m}$ de sal con un peso específico de $2,27 \mathrm{t} / \mathrm{m}^{3}$ y $42 \mathrm{~m}$ rute con un peso específico de 1,9 ton/ $\mathrm{m}^{3}$. (Ver Figura 12).

$\sigma_{v}=167,55$ ton $/ \mathrm{m}^{2}=\mathbf{1 , 6 4} \mathrm{MPa}$.

$\sigma_{h}=0,20 \mathrm{MPa}$

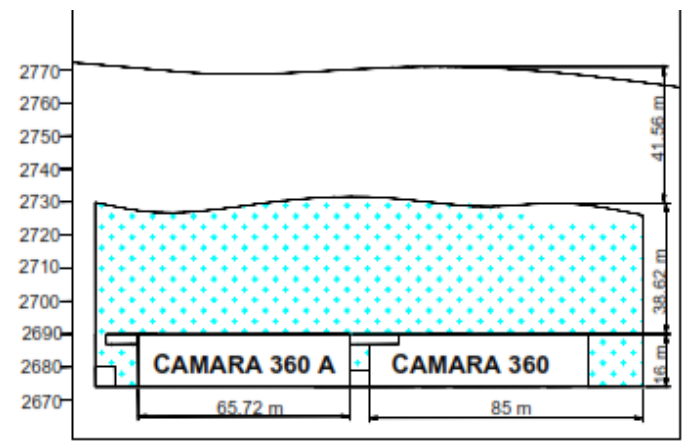

Figura 12. Corte geológico E-W sobre cámara 360. Fuente: Colsalminas (2010-2011).

\subsection{Estado tensional en cámaras}

El estado tensional de la antigua explotación, como de la catedral, se evalúa mediante la teoría del área tributaria, según la cual el esfuerzo vertical $\sigma_{v}$ aplicado sobre el machón consta no solo de la carga correspondiente al área de esta, sino de la carga de la mitad del techo de cada cámara a lado y lado del mismo. El esfuerzo sobre la columna o machón se calcula a través de (14).

$$
\sigma_{c, m}=\sigma_{v} \frac{A T}{A_{C, m}}
$$

Donde, $A T$ corresponde a 1 área tributaria columna o machón y $A_{C, m}$ es el área de la columna o machón.

La resistencia del macizo se determina a partir de la expresión de Obert-Duvall y Wang (1976), citados por Jojoa (2010, p. 27), ver Ecuación (15).

$\sigma_{p}=\sigma_{1}\left(0,778+0,222\left(\frac{W_{p}}{h}\right)\right)$

Donde, $\sigma_{p}=$ resistencia del machón, $\sigma_{1}=$ resistencia a compresión simple de la roca $\mathrm{y}_{\mathrm{p}}, \mathrm{h}=$ ancho $\mathrm{y}$ alto del machón.

\subsubsection{Estado tensional antigua explotación.}

Antiguamente el nivel Fabricalta se explotaba por el método de cámaras largas con ancho de cámara y machón de $10 \mathrm{~m}$ y una longitud constante e igual para los dos.

$$
\begin{gathered}
A T m=L c *(W p+W o) \\
A m=L c * W p
\end{gathered}
$$

Aplicando las expresiones (14), (16) y (17) se obtiene.

$$
\begin{gathered}
\sigma_{m}=1,64 M P a \frac{(10 m+10 m)}{10} \\
\boldsymbol{\sigma}_{\boldsymbol{m}}=\mathbf{3}, \mathbf{3 0} \mathbf{M P a}
\end{gathered}
$$

Al evaluar la resistencia del machón mediante la expresión de Obert- Duvall y Wang (ver ecuación 15) se obtiene $\sigma_{p}$.

$$
\begin{gathered}
\sigma_{p}=22,02 M P a \\
\boldsymbol{\sigma}_{\boldsymbol{p}}=\mathbf{2 0 , 1 8 M \boldsymbol { p a }}
\end{gathered}
$$


Entonces, el factor de seguridad es definido por la relación entre el esfuerzo y la resistencia del machón por la expresión (16). $\mathbf{F S}=\mathbf{6}$.

$$
F S=\frac{\sigma_{p}}{\sigma_{m}}
$$

\subsubsection{Estado tensional catedral.}

Aplicando la teoría del área tributaria. (Ver Figura 13).

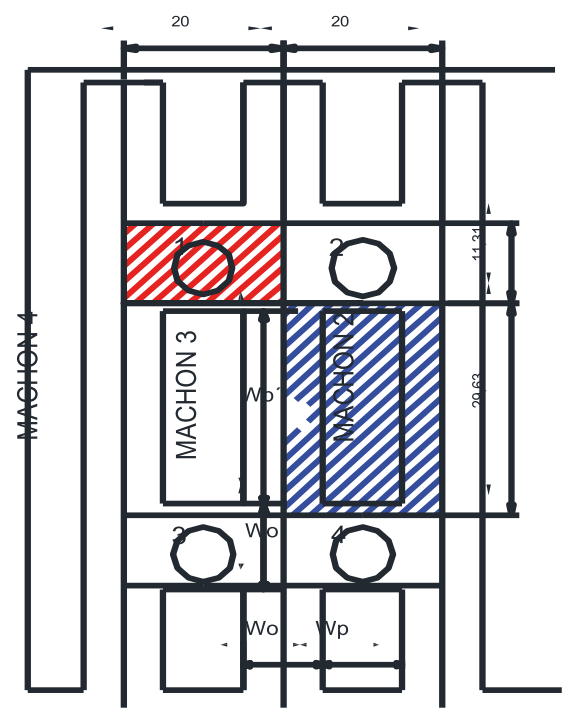

Figura 13. Área tributaria machones y columnas.

$$
\begin{gathered}
A T_{\text {columna }}=11,31 \mathrm{~m} * 20 \mathrm{~m} \\
\boldsymbol{A T}_{\text {columna }}=\mathbf{2 2 6}, \mathbf{2 \mathbf { m } ^ { \mathbf { 2 } }}
\end{gathered}
$$

Diámetro columnas 7,6- 7,9 m

$$
\begin{gathered}
\boldsymbol{A}_{\text {columna }}=\mathbf{4 5}, \mathbf{3 6} \mathbf{m}^{\mathbf{2}} \\
A T_{\text {machon }}=29,63 \mathrm{~m} * 20 \mathrm{~m} \\
\boldsymbol{A T}_{\text {machon }}=\mathbf{5 9 2}, \mathbf{6} \mathbf{m}^{\mathbf{2}}
\end{gathered}
$$

Longitud machón $=27 \mathrm{~m}$ $\mathrm{Wp}=10 \mathrm{~m}$

$$
A_{\text {machon }}=270 \mathrm{~m}^{2}
$$

Con los datos anteriores y aplicando las ecuaciones (14) a (16), se define la estabilidad de la excavación mediante el factor de seguridad (ver Tabla 6).
Tabla 6. Factor de seguridad machones y columnas.

\begin{tabular}{lccc}
\hline & $\begin{array}{c}\text { Esfuerzo } \\
(\mathrm{MPa})\end{array}$ & $\begin{array}{c}\text { Resistencia } \\
(\mathrm{MPa})\end{array}$ & FS \\
\hline Columna & 8,12 & 15,77 & 1,90 \\
Machón & 3,60 & 20,18 & 5,60 \\
\hline
\end{tabular}

Al simular el retiro de las columnas, la carga que era soportada por esta sería distribuida sobre los machones aledaños. (Ver Figura 14).

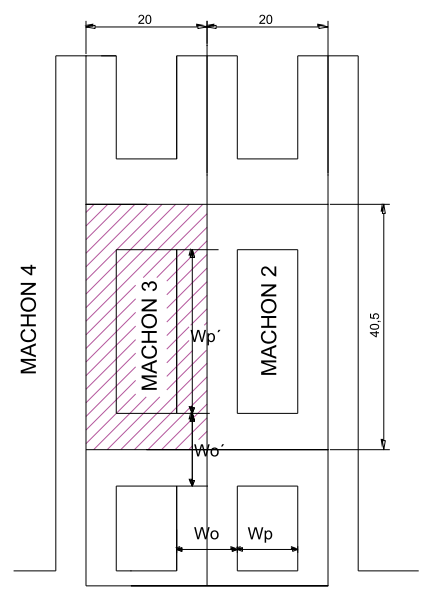

Figura 14. Carga sobre machones sin columnas según el área tributaria.

$$
\begin{gathered}
A T_{\text {machon }}=40,5 \mathrm{~m} * 20 \mathrm{~m} \\
\boldsymbol{A T}_{\text {machon }}=\mathbf{8 1 0 \mathbf { m } ^ { 2 }} \\
\boldsymbol{A}_{\text {machon }}=\mathbf{2 7 0} \mathbf{m}^{\mathbf{2}}
\end{gathered}
$$

Entonces, mediante la ecuación (14) se obtiene que $\sigma_{m}=$ 4,92 MPa.

La resistencia del machón es de 20,18 MPa y la carga sobre él sería de $4,92 \mathrm{MPa}$, con esto se obtiene un factor de seguridad de 4,1.

\subsection{Esfuerzos inducidos sobre columnas, machones y techo}

Se usó el programa Phase 5.0, el cual se basa en el cálculo de elementos finitos en dos dimensiones para determinar el estado de esfuerzos y deformaciones alrededor de excavaciones subterráneas. Para ello se usaron dos perfiles, uno longitudinal (B-B') sobre el machón de la cámara 360 y uno transversal (C-C'). 


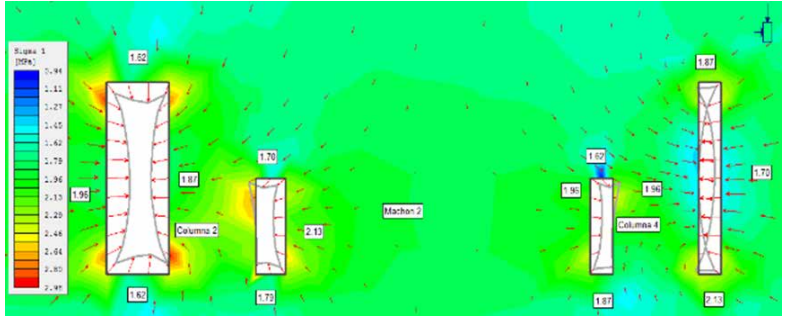

Figura 15. Perfil longitudinal B-B' concentración de esfuerzo principal mayor $\left(\sigma_{1}\right)$. Resultados estudio, análisis de datos programa Phase.

La máxima concentración de esfuerzos principal mayor se presenta en el techo y piso, especialmente en las esquinas de la excavación con valores entre 2 y $3 \mathrm{MPa}$.

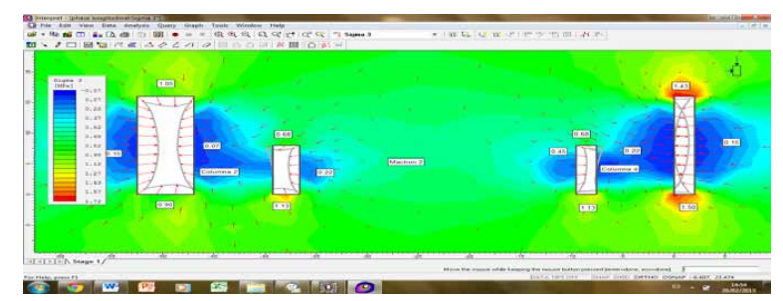

Figura 16. Perfil longitudinal B-B' concentración de esfuerzo principal menor $\left(\sigma_{3}\right)$. Resultados estudio, análisis de datos programa Phase.

Las concentraciones de esfuerzo en las paredes presentan valores bastante bajos llegando a ser negativos, lo que indica la presencia de esfuerzos a tracción que pueden conllevar la falla por cortante.

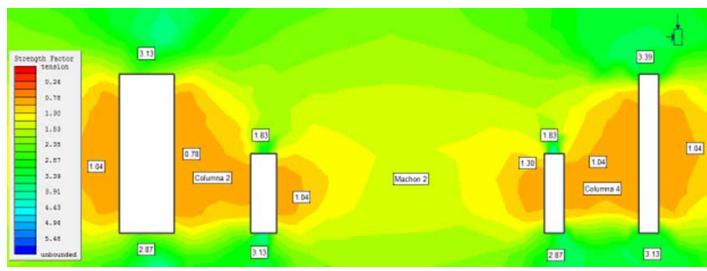

Figura 17. Perfil longitudinal B- B' concentración factor de seguridad.

Fuente: resultados estudio, análisis de datos programa Phase.

Las zonas rojizas representan el área en la cual los esfuerzos principales generados, tanto mayores como menores, están por encima de los valores de la curva de equilibrio límite. Por lo anterior, es en estos sectores en los que aumenta la probabilidad de falla con valores entre $0,7-1$.

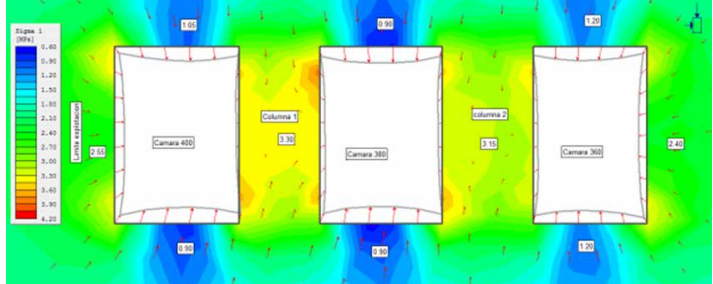

Figura 18. Perfil transversal C-C' concentración de esfuerzo principal mayor $\left(\sigma_{1}\right)$.

Fuente: resultados estudio, análisis de datos programa Phase.

Las mayores concentraciones de esfuerzo principal mayor se presentan en las paredes (2-4 MPa), especialmente en las columnas de la excavación, ya que estas tienen mayor altura.

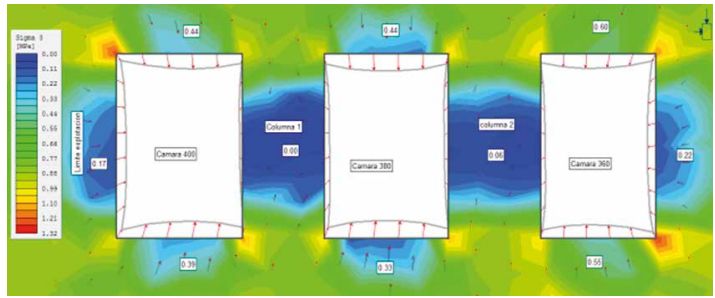

Figura 19. Perfil transversal C-C' concentración de esfuerzo principal mayor $\left(\sigma_{3}\right)$.

Fuente: resultados estudio, análisis de datos programa Phase.

Los valores menores $\sigma_{3}$ se presentan en las columnas, paredes y techo de cámaras, lo que indica una gran disminución del confinamiento propio del macizo.

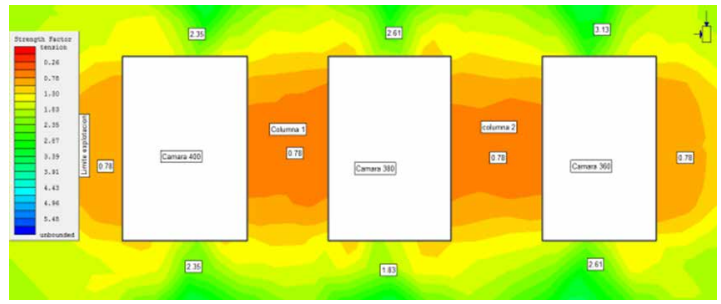

Figura 20. Perfil transversal C-C' strange factor Fuente: resultados estudio, análisis de datos programa Phase.

Se manifiesta un comportamiento similar al del perfil A-A'.

Basados en el análisis se pueden hacer las siguientes afirmaciones: i) En general, las flechas rojas indican 
la dirección de desplazamiento de la roca, siendo esta hacia el interior de la excavación; ii) La concentración de esfuerzos $\left(\sigma_{1)}\right.$ es mayor en las paredes de cada cámara; iii) El esfuerzo $\left(\sigma_{3}\right)$ presenta valores muy bajos llegando a ser negativo, lo que indica esfuerzos de tracción y bajo confinamiento que favorecen la generación de fracturas; iv) De acuerdo con la herramienta strange factor (factor de seguridad), los esfuerzos inducidos en relación con la resistencia obtenida en la curva de equilibrio que arroja el programa Roclab, son mayores, y esto se refleja en los bajos factores de seguridad obtenidos.

\subsection{Esfuerzos sobre planos de discontinuidad}

Para determinar los esfuerzos que actúan a lo largo de las paredes de la discontinuidad presentes en la roca es necesario cuantificar las presiones normales y cortantes que actúan sobre ella. (Ver Figura 21).

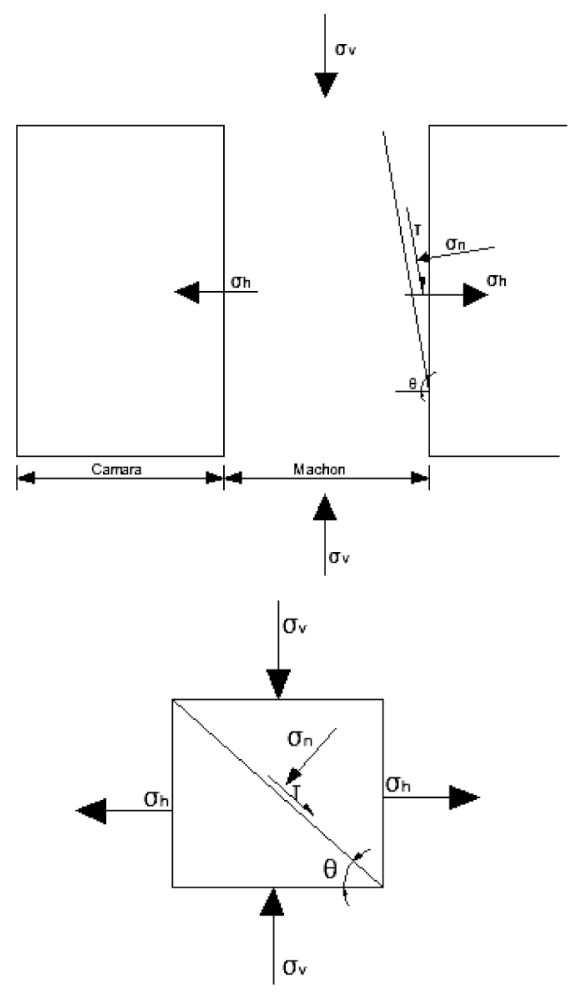

Figura 21. Esfuerzos sobre un plano de discontinuidad.

Para definir la resistencia al corte se usó el criterio de Barton y Choubey (González, 2002, pp.181-187), ver Ecuación (18). Este es un modelo empírico que permite estimar la resistencia al corte en discontinuidades rugosas y se define por la expresión.

$$
\begin{array}{r}
\tau=\sigma_{n} \tan \left[J R C \log \left(\frac{J C S}{\sigma_{n}}\right)\right. \\
\left.+\emptyset_{r}\right]
\end{array}
$$

Donde,

$\tau$ : resistencia al corte a lo largo de la discontinuidad.

$\emptyset_{r}$ : ángulo de fricción residual de la roca.

$J R C$ : coeficiente de rugosidad de la discontinuidad.

$J C S$ : resistencia a compresión de las paredes de la discontinuidad.

$\sigma_{n}$ : esfuerzo normal actuante sobre el plano de discontinuidad.

El esfuerzo normal actuante $\sigma_{n}$ es calculado mediante la expresión (19).

$$
\begin{gathered}
\sigma_{n}=\frac{1}{2}\left(\sigma_{v}+\sigma_{h}\right)+\frac{1}{2}\left(\sigma_{v}-\sigma_{h}\right) \cos 2 \theta \\
\tau=\frac{1}{2}\left(\sigma_{v}-\sigma_{h}\right) \operatorname{sen} 2 \theta
\end{gathered}
$$

Los esfuerzos normales y cortantes definidos en las ecuaciones (17) y (19) para la 'Familia 1', 'Familia 2' y estratificación se muestran en la Tabla 7.

Tabla 7. Esfuerzos normales y cortantes sobre planos de orientación preferente.

\begin{tabular}{ccccc}
\hline Plano & Orientación & $\begin{array}{c}2 \Theta \\
\left({ }^{\circ}\right)\end{array}$ & $\begin{array}{c}\sigma n \\
(\mathrm{Mpa})\end{array}$ & $\begin{array}{c}\tau \\
(\mathrm{Mpa})\end{array}$ \\
\hline Familia 1 & $80 / 168$ & 160 & 0,24 & 0,25 \\
Familia 2 & $80 / 30$ & 160 & 0,24 & 0,25 \\
Estratificación & $13 / 104$ & 26 & 1,56 & 0,32 \\
\hline
\end{tabular}

La resistencia al corte a lo largo de las discontinuidades se definió mediante la ecuación y los parámetros $\mathrm{JRC}=$ $6-8=7, J C S=11,10 \mathrm{MPa} \mathrm{y} \emptyset_{r}=38^{\circ}$.

La Tabla 8 Resistencia de discontinuidades mediante el criterio de Barton y Choube para la Familia 1 la Familia 2 y la Estratificación.

Tabla 8. Resistencia de discontinuidades mediante el criterio de Barton y Choubey.

\begin{tabular}{cc}
\hline Plano & Resistencia al cortante $(\mathrm{MPa})$ \\
\hline Familia 1 & 0,30 \\
Familia 2 & 0,30 \\
Estratificación & 1,50 \\
\hline
\end{tabular}

Fuente: resultados de estudio. 
El factor de seguridad para los planos de discontinuidad está definido por la expresión (20).

$$
F S=\frac{\text { resitencia cortante }}{\text { esfuerzo cortante }}
$$

El factor de seguridad para las Familias 1 y 2 es igual a 1,2 y para la estratificación es 4,7 , debido a que los planos de discontinuidad son casi verticales y su resistencia al corte es muy baja.

\section{ANÁLISIS DE LOS FACTORES}

En la realización de una excavación en un macizo rocoso hay una serie de factores tanto iniciales como constructivos que determinan de manera definitiva su resistencia y estabilidad a largo plazo. Dentro de estos tenemos el método de avance, las propiedades mecánicas de la roca, el método de explotación, la incidencia de excavaciones aledañas, el estado de esfuerzos y la condición de discontinuidades presentes en el macizo rocoso.

\subsection{Método de avance}

La explotación inicialmente se realizó mediante cámaras largas con $\mathrm{Wo}=10 \mathrm{~m}, \mathrm{Wp} 10 \mathrm{~m}, \mathrm{Lc}=85 \mathrm{~m}, \mathrm{H}=16 \mathrm{~m}$.

El método de arranque usado era perforación y voladura, entonces, parte de la energía liberada en una voladura se transmite directamente al medio rocoso circundante en la forma de ondas de tensión. Los movimientos asociados que se observan en un punto determinado dependen de la carga de explosivo detonado, de la distancia entre los puntos de explosión y observación y de las características de transmisión del macizo rocoso (Stagg \& Zienkiewicz, 1970, p. 206).

Cuando la intensidad de la onda de choque supera la resistencia dinámica a la compresión, se produce una trituración de la roca circundante a las paredes del barreno por colapso de la estructura intercristalina (ITGE, 1986, p. 21).

Por otra parte, la porosidad es otro factor de gran influencia, ya que la porosidad de formación provoca dos efectos: i) la atenuación de la energía de onda de choque y ii la resistencia dinámica a la compresión y consecuentemente, incremento de la trituración y porcentaje de finos.

También hay que considerar que todas las rocas en la naturaleza presentan algún tipo de discontinuidad, y los labios de estas discontinuidades son superficies planas sobre las cuales se reflejan las ondas de choque atenuando y dispersando la energía desarrollada.

\subsection{Geometría de la zona de trabajo}

La topografía superficial de la zona se caracteriza por bajas pendientes, manteniéndose un recubrimiento homogéneo en el área de estudio entre 80 y $90 \mathrm{~m}$, de los cuales $40 \mathrm{~m}$ corresponden a halita de calidades variables.

Sobre las cámaras objeto de estudio no se realizó explotación del nivel Potosí ni Guasa, por lo tanto, estas cámaras están sometidas únicamente a las sobrecargas generadas por la extracción del nivel Fabricalta.

Además vale resaltar que la cámara 400 está ubicada hacia un límite de la explotación, por lo tanto el machón es de mayores dimensiones; puede considerarse como un pilar barrera.

Las cámaras 360, 380 y 400 se realizaron con una dirección de avance W-E, mientras que las cámaras aledañas se avanzaron de $\mathrm{N}-\mathrm{S}$, esto genera una mejor distribución de los esfuerzos.

Aunque los pozos de lixiviación se encuentran en el mismo nivel, la distancia entre estos y las cámaras de la catedral son de casi $400 \mathrm{~m}$, y estos fueron diseñados para tener un diámetro máximo de 100 , lo que hace que su incidencia sea casi nula.

Debido a la forma rectangular de la excavación, pueden presentarse en el techo y el piso de la excavación grietas por tensión, pero estas no tienen un efecto importante sobre la estabilidad.

El debilitamiento al corte se inicia en las esquinas de la excavación a niveles de esfuerzos aplicados relativamente bajos (Hoek \& Brown, 1985, p. 263). La relación de esbeltez se obtiene a través de la expresión (21) 


$$
\text { Relación esbeltez }=\frac{\text { ancho }}{\text { alto }}
$$

La Tabla 9 contiene la relación de esbeltez obtenida para las columnas y machones.

Tabla 9. Relación de esbeltez columnas y machones.

\begin{tabular}{lcccc}
\hline & $\begin{array}{c}\text { Perímetro } \\
(\mathbf{m})\end{array}$ & $\begin{array}{c}\text { Diámetro } \\
(\mathbf{m})\end{array}$ & $\begin{array}{c}\text { Altura } \\
(\mathbf{m})\end{array}$ & $\begin{array}{c}\text { Relación } \\
\text { esbeltez }\end{array}$ \\
\hline Columna 1 & 23.54 & 7.49 & 16 & 0,47 \\
Columna 2 & 25.10 & 7.99 & 16 & 0,50 \\
Columna 3 & 24.66 & 7.85 & 16 & 0,49 \\
Columna 4 & 24.81 & 7.90 & 16 & 0,49 \\
Machones & - & - & 16 & 0,63 \\
\hline
\end{tabular}

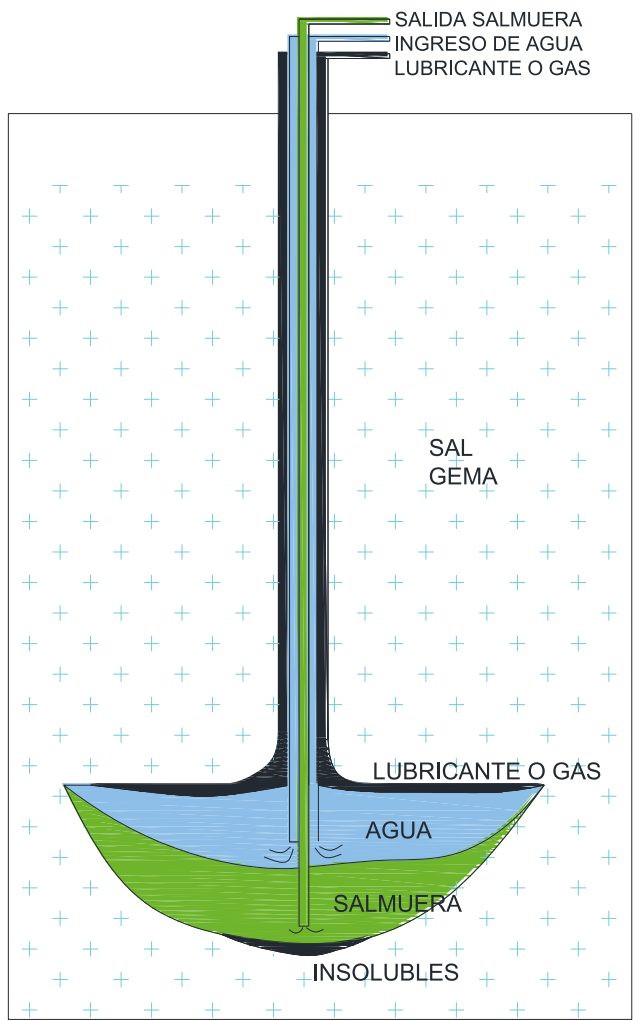

Figura 22. Sistema de explotación por lixiviación in situ. Fuente: guía de operaciones básicas en el área salinífera de Zipaquirá (Colsaliminas, 2010).

La esbeltez de un pilar está directamente relacionada con el confinamiento de este, para este caso se determina una relación aproximada de 0,5 , lo que permite definir que el nivel de esfuerzos verticales sobre los machones o columnas es mayor que el de esfuerzos horizontales, pudiendo aparecer esfuerzos de tracción en el centro del machón.

Por otra parte, una relación de 0,5 es buena, ya que con ella se garantiza que no se generen esfuerzos de pandeo tanto en machones como en columnas.

\subsection{Formación de cuñas}

Las cuñas se forman por la intersección de tres o más planos, en este caso se presentan dos familias, las cuales al intersectarse con la pared de las cámaras generan cuñas potencialmente inestables (Ver Figura 23).

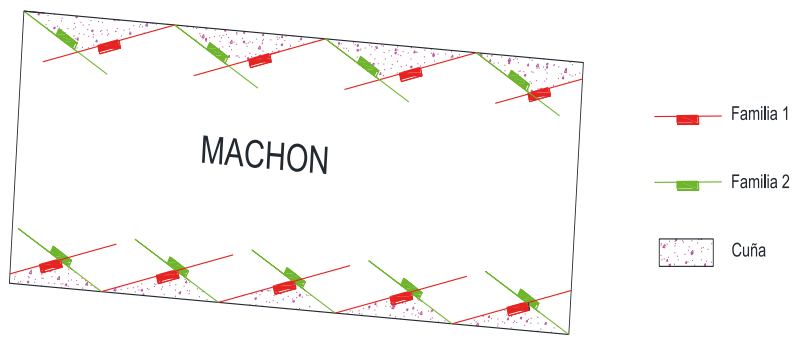

Figura 23. Posibles cuñas formadas (vista superior).

Fuente: resultados del estudio.

Para definir parámetros de la cuña se usó el programa Unwedge y criterio de Barton y Choubey.

Para la cuña pared norte cámara se tiene un $\mathrm{FS}=0.484$, Volumen $=4355 \mathrm{~m} 3$, Peso $=0,096 \mathrm{MN}$, y Altura $=9,65 \mathrm{~m}$.

Y para la cuña pared sur cámara, $\mathrm{FS}=$ estable, Volumen= $4355 \mathrm{~m} 3$, Peso $=0,096 \mathrm{MN}$ y Altura $=9,65 \mathrm{~m}$

\subsection{Propiedades mecánicas}

Se determina que las cámaras objeto de estudio están compuestas por sal, cuyo comportamiento es dúctil. En el caso de la sal se presenta un comportamiento reológico, sufriendo procesos tiempo-dependientes de fluencia o creep (aumento de las deformaciones bajo esfuerzos constantes) y de relajación (disminución de la resistencia bajo deformaciones constantes).

En el caso de la sal, el comportamiento creep presenta deformaciones lentas y continuas, tiempo dependientes, influyendo también el contenido de humedad. 
5.4.1 Medidas de convergencia en cámaras principales de la catedral

Las medidas de convergencia en las cámaras 360,380 y 400 se realizan en tres secciones por cada una, definidas de forma permanente.

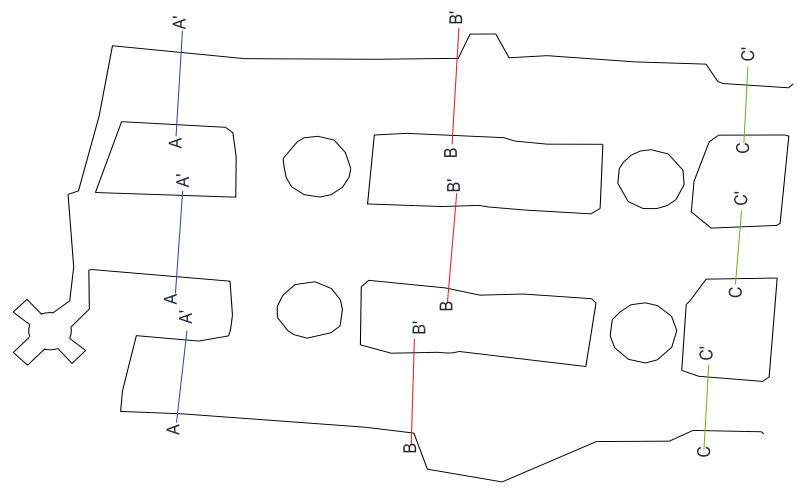

Figura 24. Ubicación secciones de control y medición.

El procedimiento consiste en medir con un flexómetro la distancia desde la pared de cada cámara en los puntos definidos en cada pared hasta un hilo de nylon que cuelga desde el techo y que se encuentra de manera permanente en las cámaras para evitar desviaciones en la medición.

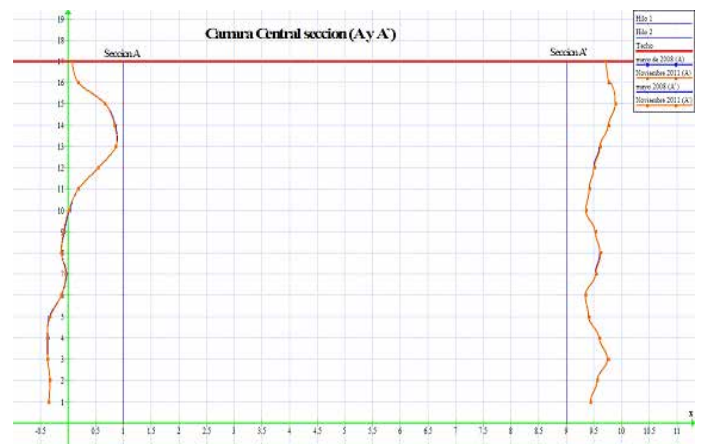

Figura 25. Medición de convergencias cámara 380 (central). Fuente: Colsalminas (2011).

Para el estudio de datos se tomaron los promedios de las deformaciones totales y su variación mensual en cada sección de las tres cámaras.

En la cámara 360 (ver Figura 26) es donde se observan las mayores deformaciones entre 0,037 y 0,032 promedio total, hecho que define una convergencia de aproximadamente el $0.4 \%$ total desde que se iniciaron las mediciones, con una velocidad de deformación de $0,000028 \mathrm{~m} /$ día.

En las cámaras 380 y 400 se presentan divergencias, pero con valores de variación muy bajos, inferiores a 0,005 $\mathrm{m}$ de deformación promedio total, con una velocidad de deformación de $-0.0000025 \mathrm{~m} /$ día.

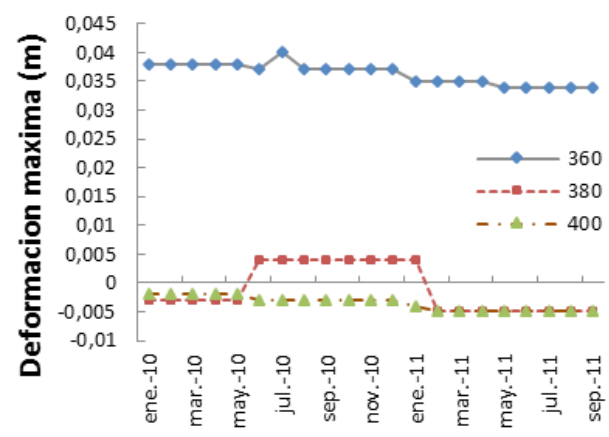

Mes controlado

Figura 26. Deformaciones máximas promedio mensual.

Basados en los datos se define que la roca mantiene un nivel de deformaciones constante (baja variación en los promedios mensuales). Pero, actualmente, tanto en machones como en columnas se están presentando nuevas fisuras que indican que la roca está llegando a su límite de resistencia.

\subsubsection{Estado tensional}

Referente al capítulo 3, las cámaras presentan un recubrimiento de aproximadamente $80 \mathrm{~m}$, lo cual implica un bajo nivel de esfuerzos tanto verticales como horizontales.

Sin embargo, los esfuerzos generados en las paredes por la realización de la excavación sobrepasan los límites de equilibrio del macizo rocoso, como se muestra en el capítulo 3.

\subsubsection{Análisis columna 2}

En el costado norte de la columna 2 se observa una cuña generada por la intersección de la columna con una diaclasa perteneciente a la familia 2, lo mismo ocurre en el costado sur donde se presenta una diaclasa perteneciente a la familia 1 . 
Tabla 10. Características columna 2

\begin{tabular}{|c|c|c|c|c|}
\hline & \multicolumn{4}{|c|}{$\begin{array}{l}\text { CARACTERÍSTICAS GENERALES DE LA } \\
\text { COLUMNA } 2\end{array}$} \\
\hline $\begin{array}{l}\text { Altura } \\
\text { columna }\end{array}$ & $\begin{array}{l}\text { Diámetro } \\
\text { columna }\end{array}$ & $\begin{array}{l}\text { Tipo de } \\
\text { sal }\end{array}$ & \%de sal & Insolubles \\
\hline 16 & 7 & S2 & 70 & $30 \%$ \\
\hline
\end{tabular}

Fuente: datos de estudio.

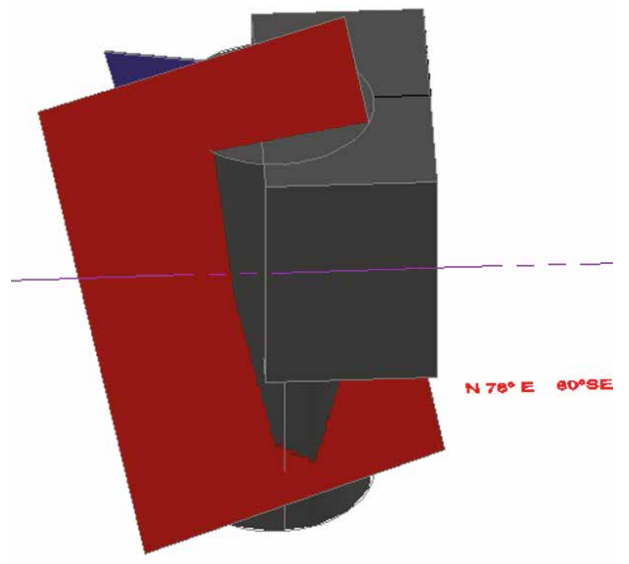

Figura 27. Incidencia de discontinuidades dentro de la columna 2.

A partir de los análisis realizados se determina que las fracturas se deben principalmente a cargas verticales, ya que estos machones se encuentran en un estado de no confinamiento, creando un estado de relajación en las paredes de la columna.

Los núcleos usados para compresión simple asemejan el dimensionamiento de las columnas a una escala de 1:160, $\mathrm{y}$ al ser falladas, los tipos de fracturas generadas fueron similares a las observadas en las cámaras (fracturas verticales fenómeno y de barril).

La perforación realizada a la columna 2 se definió RQD de 94,62, lo cual indica que la roca es de buena calidad. Y observando las fracturas a lo largo de la perforación se concluye que este diaclasamiento presentado en las cámaras no ha afectado el núcleo de la columna.

\section{ESTABILIZACIÓN DE ZONAS AFECTADAS}

\subsection{Sistema de estabilización}

Para el sistema de explotación de cámaras largas implementado en la mina de sal de Zipaquirá definió que un sistema con pernos de anclaje ofrece más ventajas, debido a que:

i. Orientación y separación de diaclasas genera grandes bloques.

ii. Por la posición de los estratos.

iii. Comportamiento elastoplástico de la roca.

iv. Genera una mayor resistencia al corte a lo largo de las paredes de la cuña.

v. Genera una adherencia del material suelto con el macizo rocoso.

vi. Al ser un método de sostenimiento puntual se puede adecuar a las necesidades de las cámaras.

vii. Genera mejor distribución del peso de la cuña.

viii. Fácil instalación.

ix. Facilidad en el diseño y modelamiento.

Además, estos minimizan las deformaciones inducidas por el peso muerto de la roca aflojada, así como también aquellas inducidas por la redistribución de los esfuerzos en la roca circundante a la excavación.

\subsection{Diseño de pernos}

Para calcular los esfuerzos generados por la cuña sobre el perno se usa el diagrama de cuerpo libre presentado en la Figura 28.

$N_{\alpha}=W \cos \alpha$

$T_{\alpha}=W \operatorname{sen} \alpha$

$R_{s}=N_{\alpha} \tan \emptyset=W \cos \alpha \tan \emptyset$

$W c=\frac{(n \operatorname{sen} \alpha-\cos \alpha \tan \emptyset) W}{\cos (\alpha+\beta)+\operatorname{sen}(\alpha+\beta) \tan \emptyset}$ 


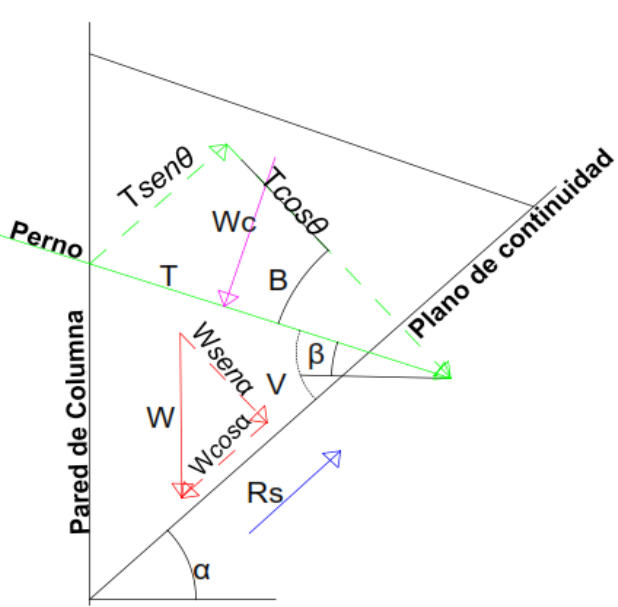

Figura 28. Diagrama de fuerzas perno-cuña.

A continuación se relacionan los parámetros de la figura 28 con su respectiva denominación.

Wc : peso muerto de los bloques separados por las superficies agrietadas (ton)

$\phi$ : $\quad$ ángulo de fricción de la grieta

$\mathrm{R}_{\mathrm{s}}$ : $\quad$ fuerza de fricción (ton)

n: $\quad$ factor de seguridad

$\mathrm{N}_{\alpha}$ : fuerza normal a la superficie de la discontinuidad debido al peso muerto del bloque

$\mathrm{T}_{\alpha}$ : fuerza que provoca el movimiento (ton)

W: peso del bloque

$\alpha$ : $\quad$ ángulo de la grieta

$\beta$ : $\quad$ ángulo del perno con respecto a la horizontal

Para calcular la resistencia del perno se usaron las expresiones (25), (26) y (27).

$$
\begin{gathered}
R \max =\sigma_{a} A \\
R=\frac{R \max }{n} \\
A=\frac{\pi D^{2}}{4}
\end{gathered}
$$

Donde,

A: $\quad$ área del perno $\left(\mathrm{cm}^{2}\right)$

T: $\quad$ Rmax=capacidad máxima de soporte del perno a tracción $(\mathrm{kg})$
$R: \quad$ fuerza axial permitida en el perno $(\mathrm{kg})$

$\sigma_{a}: \quad$ esfuerzo en el límite elástico

D: $\quad$ diámetro del perno

El espaciamiento de los pernos está estrechamente relacionado con la longitud de los mismos, entonces es espaciamiento entre pernos $b$ está en función de la longitud como se expresa en la ecuación (29).

$$
b=\frac{2}{3} l
$$

$\begin{array}{ll}\text { b: } & \text { espaciamiento entre pernos }(\mathrm{m}) \\ \mathrm{l} & \text { longitud de los pernos }\end{array}$

6.2.1 Estabilización columna dos. Para la cuña de la columna 2 se determina un pernado con base en los siguientes datos ${ }^{\phi}=38^{\circ}, n=2$

$\alpha=60^{\circ}, \beta=25^{\circ}, \mathrm{W}=16.98$ ton. Mediante la aplicación de las ecuaciones (22) a (24) se obtuvo $T \alpha=14.71$ ton, $N \alpha=8.49$ ton, y $R s=6.63$ ton.

Como $\mathrm{T}_{\alpha}$ es mayor que $\mathrm{R}_{\mathrm{s}}$ entonces existe la probabilidad de que se genere un deslizamiento o colapso de la cuña.

Wc $=26,31$ ton, que es la carga axial que incide sobre el perno.

Inicialmente un diámetro de varilla de 1"y se aplicaron las ecuaciones 25 y 26 obteniéndose los siguientes resultados:

$\sigma_{a}=2400 \mathrm{~kg} / \mathrm{cm}^{2}$

$\mathrm{D}=2,54 \mathrm{~cm}$

$\operatorname{Rmax}=12,15$ ton

$\mathbf{R}=\mathbf{6 , 0 7 7}$ ton

Teniendo en cuenta la carga axil sobre el perno $(\mathrm{Wc}=26,31)$ y la resistencia axial de cada perno $(\mathrm{R}=6,077)$, se determinó que se necesitan:

\#pernos $=\mathrm{Wc} / \mathrm{R}$

\#pernos $=5$

Se definió que la cuña se proyecta en profundidad máximo $0,6 \mathrm{~m}$ en su parte superior, por lo tanto se fijó que 
la longitud de los pernos varía de 1,3 a 1,5 m. Mediante la ecuación 28 se definió el espaciado entre pernos.

$1=1,5$

$\mathrm{b}=\mathbf{1 , 0} \mathbf{m}$

Para la cuña norte de la columna 2 se puede aplicar el mismo diseño, ya que son similares en sus características.

\subsection{Estabilización de machones.}

Para el diseño del sostenimiento en machones se tomaron los datos de la cuña que arroja el programa unwedge.

$\mathrm{W}=0,094 \mathrm{MN}=9,4$ ton

$\phi=38^{\circ}$

$\mathrm{n}=2$

$\alpha=80^{\circ}$

$\beta=25^{\circ}$.

Mediante la aplicación de las ecuaciones 21 a 23 se definieron los siguientes parámetros:

$T_{\alpha}=925$ ton

$N_{\alpha}^{\alpha}=1,63$ ton

$R_{s}=1,27$

$\mathbf{W c}=\mathbf{3 4 , 7 5}$ ton, que es la carga axial que incide sobre el perno.

Inicialmente, un diámetro de varilla de 1" y se aplicaron las fórmulas de 26 y 27 obteniéndose los siguientes resultados:

$\sigma_{a}=2400 \mathrm{Kg} / \mathrm{cm}^{2}$

$\mathrm{D}=2,54 \mathrm{~cm}$

$\operatorname{Rmax}=12,15$ ton

$\mathrm{R}=\mathbf{6 , 0 7 7}$ ton

Teniendo en cuenta la carga axil sobre el perno $(\mathrm{Wc}=26,31)$ y la resistencia axial de cada perno ( $\mathrm{R}=6,077)$ se determinó que se necesitan:

$\#$ pernos $=\mathrm{Wc} / \mathrm{R}$

\#pernos $=5$
Se definió que la cuña se proyecta en profundidad máximo 1 en su parte superior, por lo tanto se fijó que longitud de los pernos varía de 1,5.

\section{CONCLUSIONES}

Mediante el levantamiento de discontinuidades en los machones de las cámaras se determinó que presentan un comportamiento predominante caracterizado por el paralelismo con respecto al eje de la cámara, siendo esta una condición desfavorable, alto buzamiento que implica bajos esfuerzos tanto normales como cortantes a lo largo de la diaclasa, y bajo grado de meteorización, que favorece la resistencia residual de la discontinuidad.

En el análisis de propiedades físicas de la sal gema se obtuvo un peso específico de $2,25 \mathrm{KN} / \mathrm{m}^{3}$, indicando que tiene una dureza media, con una humedad natural $\mathrm{o}$ in situ de $0,07 \%$, siendo casi despreciable y no incide en la disgregación de las sales presentes en las cámaras.

En la evaluación de los resultados de compresión simple se determinó que la roca presenta un comportamiento elasto-plástico dúctil con fluencia dependiente del tiempo, ya que la resistencia pico y la residual son iguales, pero se presentaron grandes deformaciones entre una y otra.

Mediante el análisis estructural de cada familia de discontinuidades de factores de seguridad de 1,2 para diaclasas y 4,7 para la estratificación, pero, al intersectarse $\mathrm{y}$ formar cuña, la afectación del macizo es mayor y la roca pierde estabilidad.

Con el uso de programa Phase se mostró que las máximas concentraciones de esfuerzos se presentan en las paredes tanto de machones como columnas, siendo allí donde se observa el mayor deterioro, además con la herramienta Strange Factor se calculó que en algunos sectores la condición de esfuerzos iguala la resistencia del macizo rocoso, haciéndose más visible la deformación a lo largo del tiempo.

Actualmente, el dimensionamiento de cámaras es estable, pero con el tiempo puede presentar deformaciones considerables que afectan la estabilidad debido a sus características mecánicas. Sin embargo se definió que al retirar las columnas los machones resisten la nueva redistribución de cargas manteniendo la estabilidad. 
Dentro del análisis de los factores se define que el estado de esfuerzos verticales es el que genera la mayor afectación, haciéndose esto visible en la propagación y aumento de discontinuidades.

Como alternativa de sostenimiento se eligió el sistema de pernado, ya que este ofrece grandes ventajas para el método de explotación implementado en la catedral, garantizando una fijación de cuñas puntuales y un aumento de la resistencia al corte a lo largo de la discontinuidad.

\section{RECOMENDACIONES}

Debe hacerse un seguimiento, mantenimiento y control continuo de las condiciones de diaclasas, con el fin de evitar la propagación de estas en machones y el aumento de las deformaciones tanto de paredes como de techos.

Se sugiere la ubicación de un deformímetro en el interior de la perforación horizontal realizada a la columna 2 , con el fin de medir las convergencias generadas.

El control de convergencias generales de las cámaras puede realizarse con topografia, pues garantiza mayor exactitud en los datos para evitar el cerramiento de las cámaras.

El desabombe disminuye el confinamiento del núcleo de los machones y por lo tanto su capacidad portante y esbeltez, reduciendo su factor de seguridad. Debe procurarse no realizar estas prácticas, en su lugar deben implementarse medidas de sostenimiento diseñadas en el presente proyecto.

Se recomienda como alternativa de sostenimiento el uso de pernado tipo ranura y cuña, ya que tiene mayor adherencia y resistencia mitigando el deterioro de las cámaras y caída de bloques.

\section{REFERENCIAS}

Colsalminas LTDA. (2010, enero). Informes mantenimiento técnico minero de la nueva Catedral de Sal. Zipaquirá: Colsalminas LTDA.

Colsalminas LTDA. (2011, nov.). Informes mantenimiento técnico minero de la nueva Catedral de Sal. Zipaquirá: Colsalminas LTDA.

González, L. (2002). Ingeniería geológica. Madrid: Pearson Educación.

Hoek, E.(1986). Practical Rock Engineering. Canadá: EK.

Hoek, E. \& Brown, E.T. (1985). Excavaciones subterráneas en roca. México: McGraw Hill.

Hoek, E., Carranza, C. \& Corkum, B. (2002). Criterio de falla de Hoek-Brown. In North American Rock Mechanics Symposium, 5th, Proceedings. (p. 267-273) Toronto, Canada.

Instituto Tecnológico Geominero de España -ITGE(1986). Manual de perforación y voladura. España: ITGE.

Jojoa, J. W. (2010). Módulo 3 Excavaciones subterráneas múltiples, diseño geomecánico de explotaciones por cámaras y pilares. Sogamoso, Colombia: Universidad Pedagógica y Tecnológica de Colombia.

Jojoa, J. W. (2008). Mecánica de rocas aplicada a la minería. Módulo de clase. UPTC, Escuela Ingeniería de Minas.

Stagg, K.G. \& Zienkiewicz, C. (1970). Mecánica de rocas en la ingeniería práctica. Madrid: Blume. 